Running head: CHRONOTYPE AND PRIMING

\title{
Time of day affects implicit memory for unattended stimuli
}

\author{
Nicolas Rothen and Beat Meier \\ Institute of Psychology and Center for Cognition, Learning and Memory, \\ University of Bern, Bern, Switzerland
}

Original article submitted to Consciousness and Cognition (08/06/2016) Revision of article submitted to Consciousness and Cognition (12/09/2016)

Author Note

Nicolas Rothen and Beat Meier, Institute of Psychology and Center for Cognition, Learning and Memory, University of Bern, Switzerland.

Correspondence concerning this article should be addressed to Nicolas Rothen, University of Bern, Institute of Psychology, Fabrikstrasse 8, 3012 Bern, Switzerland, Phone +41(0)31631 4732, E-mail: nicolas.rothen@ gmail.com

Word count: approximately 2850 


\begin{abstract}
We investigated whether circadian arousal affects perceptual priming as a function of whether stimuli were attended or ignored during learning. We tested 160 participants on- and off-peak with regards to their circadian arousal. In the study phase, they were presented with two superimposed pictures in different colours. They had to name the pictures of one colour while ignoring the others. In the test phase, they were presented with the same and randomly intermixed new pictures. Each picture was presented in black colour in a fragment completion task. Priming was measured as the difference in fragmentation level at which the pictures from the study phase were named compared to the new pictures. Priming was stronger for attended than ignored pictures. Time of day affected priming only for ignored pictures, with stronger priming effects off-peak than on-peak. Thus, circadian arousal seems to favour the encoding of unattended materials specifically at off-peak.
\end{abstract}

KEYWORDS: chronotype, circadian arousal, time of day, memory, priming, attention 


\section{Time of day affects implicit memory for unattended stimuli}

Circadian arousal patterns affect explicit and implicit memory differentially depending on the time of testing (May, Hasher, \& Foong, 2005). Explicit memory, that is, conscious recollection of previous experiences (Graf \& Schacter, 1985), is better when tested at on-peak times of circadian arousal (Baddeley, Hatter, Scott, \& Snashall, 1970; Hasher, Chung, May, \& Foong, 2002; Intons-Peterson, Rocchi, West, McLellan, \& Hackney, 1998; May, Hasher, \& Stoltzfus, 1993; West, Murphy, Armilio, Craik, \& Stuss, 2002; Yang, Hasher, \& Wilson, 2007). In contrast, implicit memory, that is, performance facilitation in the absence of conscious recollection (Graf \& Schacter, 1985; Schacter, 1987), may be better when tested at off-peak times of circadian arousal (May et al., 2005). So far, the relationship between circadian arousal and memory performance has mainly focused on retrieval processes (Hasher et al., 2002; May et al., 2005, 1993; Yang et al., 2007). Here, we investigate the effect of circadian arousal in relation to intentional and incidental encoding. Specifically, we test whether items that were either attended or ignored during the study phase have differential effects on repetition priming in dependence of chronotype and time of day.

We expected that circadian arousal affects intentional and incidental encoding in a similar way as it affects other cognitive functions (e.g., Blatter \& Cajochen, 2007; Murray et al., 2009; Paradee, Rapport, Hanks, \& Levy, 2005; Schmidt, Collette, Cajochen, \& Peigneux, 2007). Hence, we predicted that the interplay between time of day and circadian arousal would influence the contribution of automatic and controlled processes in a priming task. Automatic processes are regarded as fast, parsimonious on processing resources and supposed to occur without conscious control. In contrast, controlled processes are slower, resource demanding and rely on conscious control (Meier, Morger, \& Graf, 2003). Thus, priming for previously attended versus ignored items should be differentially affected. 
Specifically, we hypothesized stronger priming effects for items that were attended at study than items that were ignored at study. Moreover, we expected stronger priming effects for items attended at study when tested on-peak (i.e., optimal time of day) rather than off-peak (i.e., non-optimal time of day) and stronger priming effects for items ignored at study when tested off-peak rather than on-peak.

\section{Method}

\section{Participants}

The experiment was conducted in the context of a research methods class at the University of Bern. Ten second year psychology students helped with data collection for this experiment. They recruited and tested 16 participants each. Inclusion criteria were an age range from 18-30 years and normal or corrected-to-normal vision. The study was approved by the local ethics committee of the Faculty. Participants were informed that they could withdraw at any time during the experiment before they consented to participate. A total of 160 volunteers (93 female and 67 male, age $M=22.52$ years, $S D=2.30$, range 18-29 years) participated in this study.

\section{Materials}

We selected a random subset of 184 items from a standardized set of 260 line drawings (Snodgrass \& Vanderwart, 1980). Forty items were used to create 20 stimuli of two superimposed pictures, ten of which served as primacy and ten of which served as recency stimuli. The remaining 144 items served as targets and were used to create two different lists (A and B) of 72 items each. Each list consisted of two sublists (A1 and A2 or B1 and B2) in order to create 36 stimuli of superimposed pictures. One item of the superimposed pictures was always red and the other blue. The superimposed pictures were pseudorandomly assigned to each other in order to prevent semantic overlap (Figure 1, top). The target items were also used to create a set of single pictures in black colour. Chronotype was assessed 
with the German version of the Morningness-Eveningness-Questionnaire (D-MEQ; Griefahn, Künemund, Bröde, \& Mehnert, 2001; see also, Horne \& Ostberg, 1976).
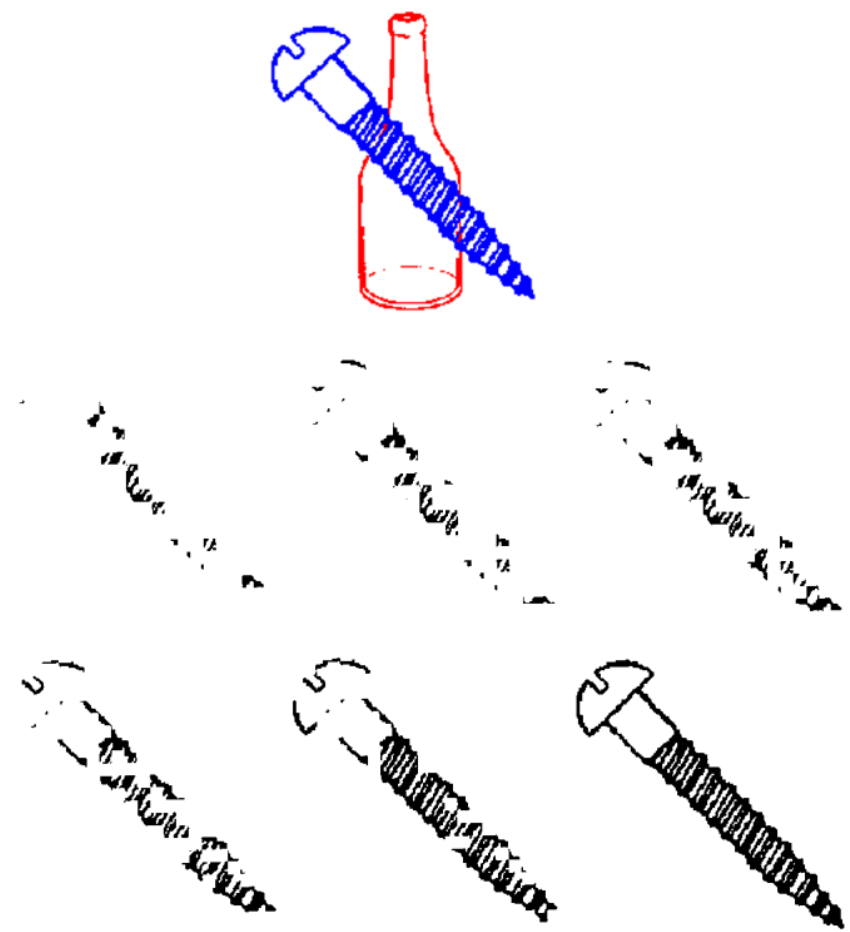

Figure 1. Example stimuli of the study (screw and bottle) and test phase (screw). Two superimposed line drawings were used as stimuli during the study phase (top). Participants were asked to name the object depicted in one colour (e.g., blue) and ignore the object depicted in the other colour (e.g., red). Fragmented objects served as stimuli for the test phase (bottom). There were always six levels of fragmentation. Each trial started with the most fragmented version, with the object becoming less fragmented until the participant was able to identify it.

\section{Procedure}

First, participants completed an online version of the D-MEQ. Then, they were assigned to the experimental condition either in the morning (between 6:00 and 10:00) or in the evening (between 17:00 and 21:00). The experimental tasks took approximately 60 minutes to complete. 
For the experiment, participants were individually tested. They were informed that they will be presented with red and blue line drawings. Depending on the condition, they were instructed to name (i.e., attended) the red line drawings and ignore (i.e., unattended) the blue line drawings or vice versa. Participants were first presented with the primacy stimuli (10 trials), then the target stimuli of one list (36 trials), and finally the recency stimuli (10 trials). The stimuli were presented in randomised order within each of these lists. The experimenter initiated each trial by a key-press after the participant named the relevant item. After the study phase, participants were required to complete an unrelated filler task of approximately 20 minutes duration.

In the test phase, participants were instructed that they will be presented with fragmented line drawings which they will have to identify (Figure 1, bottom) without reference to the study phase (cf. Meier, Theiler-Bürgi, \& Perrig, 2009). Half of the pictures of the test phase had been presented during the study phase (i.e., old items). The other half were from the list which had not been presented before (i.e., new items). The pictures were presented in randomised order. The experimenter initiated each trial by a key-press upon which the fragment of an object was presented on the screen for $5 \mathrm{sec}$, followed by a blank screen during which the subjects were required to respond. If an object was named correctly, the experimenter initiated the presentation of the next picture in its most fragmented version. If the object was named incorrectly or if the participant did not respond, the same object was presented again, but in a less fragmented form, until the participant was able to name it correctly. A total of six different levels of fragmentation were used. If an object was identified in its most fragmented version, it was scored as 1; if an object was identified in the second most fragmented version, it was scored as 2, etc. Thus, lower identification levels denote better identification performance. A mean score was calculated for each condition (old items and new items). 
Lists A and B were counterbalanced between participants and served equally often as old items and new items, respectively. Whether an item had to be ignored or named and whether it was red or blue was fully counterbalanced between the sublists (A1 and A2 or B1 and B2). Counterbalancing resulted in eight different versions which were used approximately equally often for the experimental conditions.

\section{Analysis}

The alpha level was set to .05 for all statistical analyses. Reported effect sizes denote partial eta squared $\left(\eta_{\mathrm{p}}^{2}\right)$. Priming was calculated on an individual basis according to the following equation: priming $=$ new items - old items, where old items refers to attend and unattended items, respectively (cf. Table 1 for the raw-scores). Participants were classified as morning or evening types according to the question: "One hears about 'morning types' and 'evening types'. Which one of these types do you consider yourself to be?" The potential answers were: "Definitely a morning type", "Rather more a morning type than an evening type", "Rather more an evening type than a morning type “, "Definitely an evening type" (DMEQ, question 19). We did so in order to be able to use all participants in our analyses without making an arbitrary decision about how to classify participants with a D-MEQ sum score in the neutral range. Moreover, interpretations of effects based on extreme groups would be limited (e.g., testing only morning and evening types and dismiss neutral types) and could not be generalised.

To validate our classification approach, we plotted the self-declared morning- and evening-types in different colours in relation to their D-MEQ score and explored the potential correlation between the age of the participants and their D-MEQ scores (Figure 2). The results show that participants are indeed able to correctly classify themselves as morning and evening types on the basis of a single question (i.e., question 19, D-MEQ). All participants who classified themselves as evening types according to question 19 of the D-MEQ were 
indeed evening-types according to their D-MEQ scores (i.e., < 42). All but two participants classified themselves in correspondence to their D-MEQ scores as morning types (i.e., > 58). Furthermore, participants classified themselves according to the tendency of their D-MEQ scores in the neutral range (i.e., 42-58). However, we were not able to observe a significant correlation between age and D-MEQ score, $r_{\text {Pearson }}(160)=.05, p=.572$, which might be due to the relatively small age range and the fact that only young adults were tested. Furthermore, we were also not able to observe that young adults were primarily neutral and evening types as previously suggested in some research articles (Anderson, Campbell, Amer, Grady, \& Hasher, 2014).
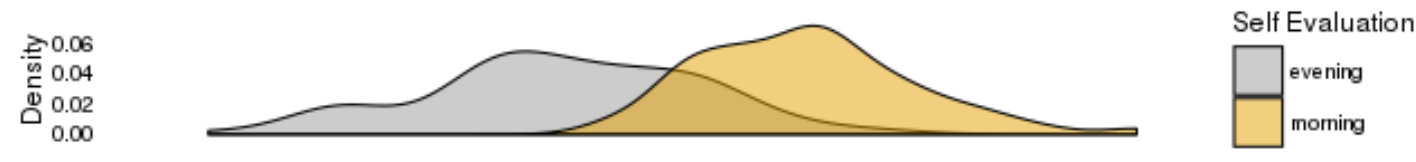

30

40

50

60

70

28

26

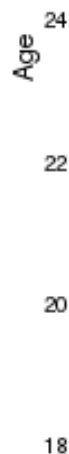

18
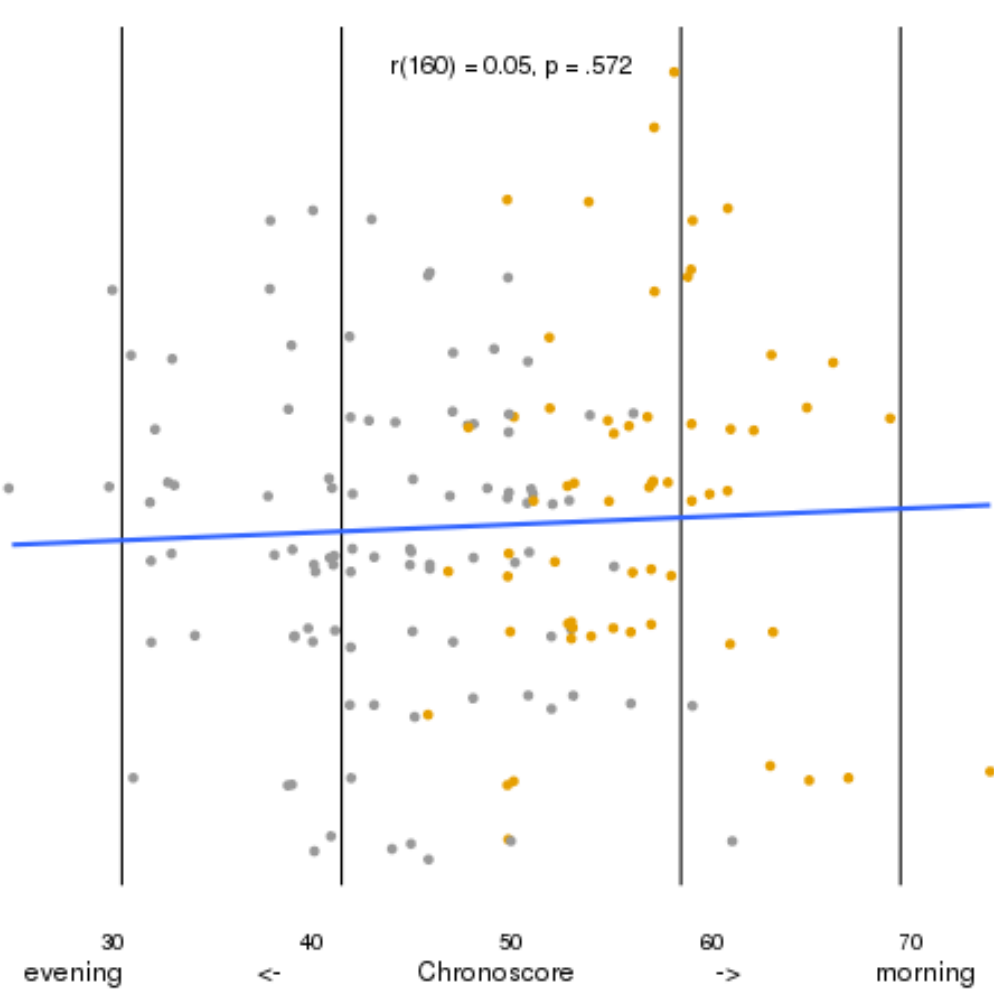

$\stackrel{60}{->}$
70 morning

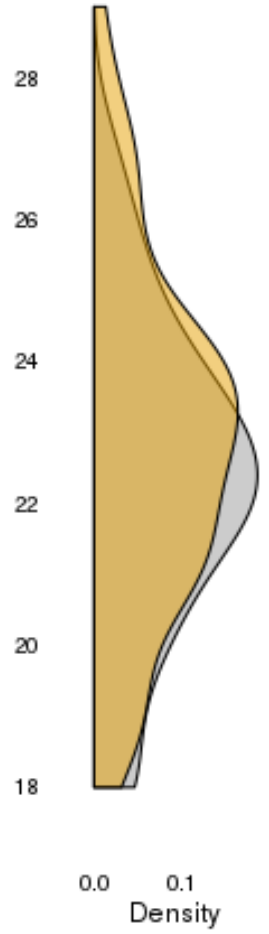


Figure 2. Participants who classified themselves as evening types are shown in grey and participants who classified themselves as morning types are shown in orange. The central panel depicts the relationship between self-declared eveningness / morningness (D-MEQ, question 19), age and the achieved score in the D-MEQ. Vertical lines indicate the thresholds for the definite evening, moderate evening, neutral, moderate morning, and definite morning range (from left to right). Note, a random jitter between $+/-0.5$ was added to each datapoint in order to help visual inspection of the data. The blue line indicates the correlation between age and D-MEQ score. The top panel depicts the density of the distribution of the D-MEQ scores. The left panel depicts the density of the distribution of the participants' age.

\section{Results}

Figure 3 shows priming for attended and unattended items as a function of time of testing (morning vs. evening) and chronotype (morning type vs. evening type). We conducted a mixed three-factorial Analysis of Variance (ANOVA) with the within subject factor Encoding (attended vs. unattended) and the between subject factors Time of Testing (morning vs. evening) and Chronotype (morning type vs. evening type). The analysis revealed a significant main effect Encoding due to more priming in the attended condition than the unattended condition, $F(1,156)=83.87, p<.001, \eta_{\mathrm{p}}{ }^{2}=.35$ and a significant triple interaction Encoding x Time of Testing x Chronotype, $F(1,156)=4.06, p=.046, \eta_{\mathrm{p}}{ }^{2}=.03$. No other effect was significant, all $F_{\mathrm{s}}(1,156)<2.53$, all $p \mathrm{~s}>.113$. Because the pattern of the results was nearly identical for morning and evening types with regards to on- and off-peak testing, we collapsed Chronotype and Time of Testing into the single factor Peak (on-peak vs. offpeak) in order to increase the statistical power of the post-hoc tests. That is, unattended stimuli showed significantly greater priming off-peak than on-peak, $t(158)=2.25, p=.026$. By contrast, the same difference was not significant for attended items, $t(158)=0.26, p=$ .792. Moreover, we tested whether priming for the unattended items was significantly 
different from zero, which was the case for off-peak testing but not for on-peak testing, $t(78)$ $=2.66, p=.009$ and $t(80)=0.44, p=.658$, respectively. Thus, priming for unattended stimuli occurred only during off-peak.

Table 1:

Descriptive statistics ( $N$ ) for participants and (mean values and standard errors in parenthesis) for old items (i.e., attended and unattended) and new items.

\begin{tabular}{lllrrr}
\hline chronotype & testing time & $\mathrm{N}$ & attended [old] & unattended [old] & new items \\
\hline morning type & morning & 31 & $2.51(.076)$ & $3.04(.088)$ & $3.01(.063)$ \\
& evening & 30 & $2.47(.065)$ & $2.77(.089)$ & $2.98(.072)$ \\
evening type & morning & 49 & $2.63(.063)$ & $2.99(.073)$ & $3.09(.050)$ \\
& evening & 50 & $2.51(.060)$ & $3.02(.074)$ & $3.01(.054)$
\end{tabular}

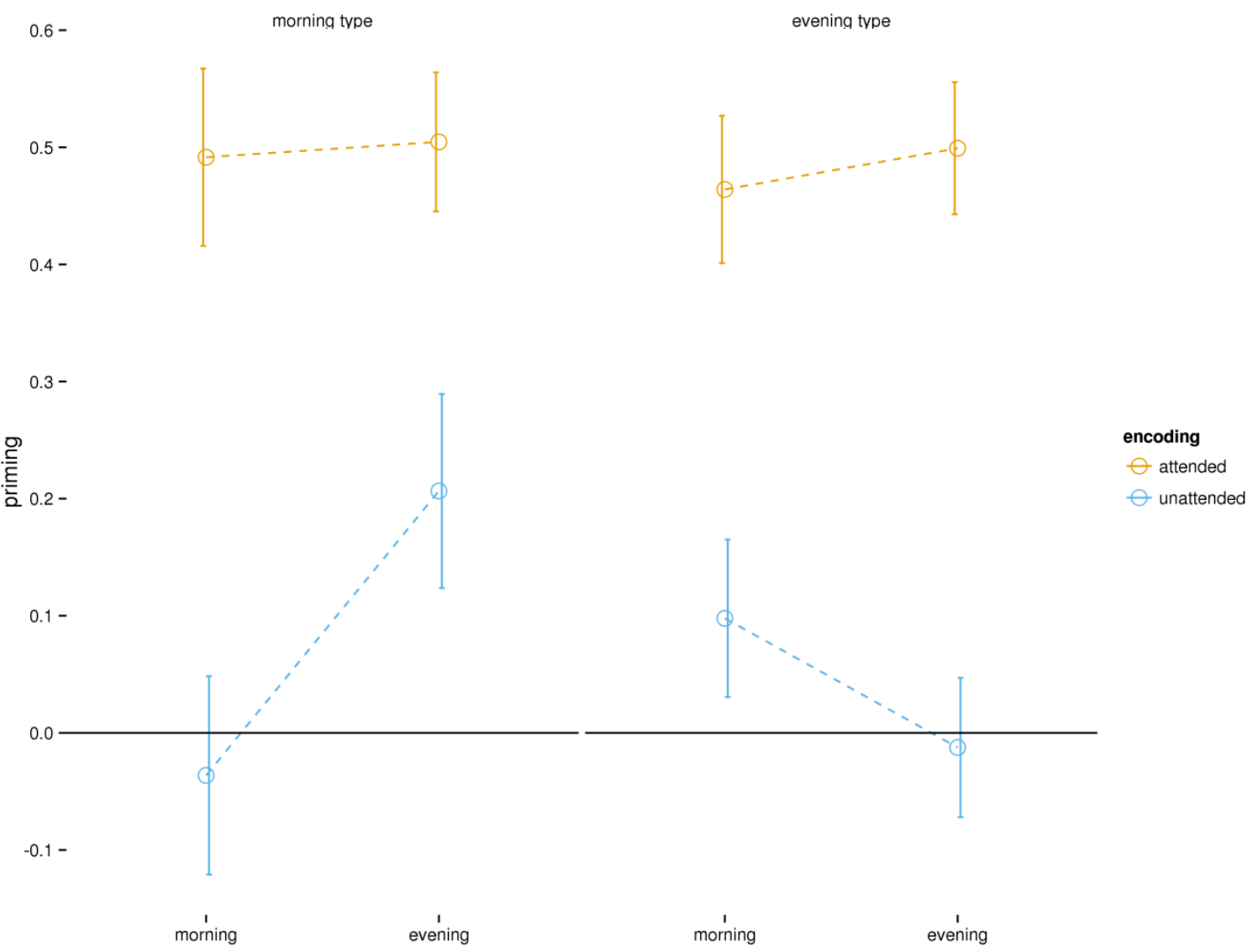


Figure 3. Priming (new items - old items) for attended and unattended items (i.e., old items) as a function of time of testing (morning vs. evening) and chronotype (morning type vs. evening type). The figure shows that priming for attended items is enhanced over priming for unattended items. Priming for attended items is not affected by the time of testing. However, priming for unattended items is affected by the time of testing. For unattended items priming only occurs when participants are tested at their non-optimal time of day. Error bars represent standard errors.

\section{Discussion}

Our findings suggest an impact of circadian arousal on encoding requirements. For pictures that were ignored during the study phase, priming was stronger when tested at the non-optimal time of the day in comparison to priming at the optimal time of the day. In fact, there was no priming at the optimal time of the day. By contrast, no difference in priming was found between optimal and non-optimal time of the day for pictures that were attended to during the study phase. Our results are in line with the notion of a performance advantage for automatic processes at the non-optimal time of the day over the optimal time of the day.

More specifically, our results are also consistent with findings suggesting that cognitive performance for implicit memory benefits from testing at off-peak times of circadian arousal (May et al., 2005). However, controlling for comparable external task characteristics between the attended and ignored condition at encoding, our findings suggest that there is a performance advantage for controlled processes over automatic processes. That is, overall priming is reduced for ignored stimuli. In contrast to our hypothesis, priming for attended stimuli was not affected by time of day as one might expect on the basis of previous research showing that cognitive performance for explicit retrieval from memory benefits from testing at peak times of circadian arousal (Baddeley et al., 1970; Hasher et al., 2002; Intons-Peterson et al., 1998; May et al., 1993; West et al., 2002; Yang et al., 2007). 
Our results further suggest that priming is not generally enhanced when participants are tested at their non-optimal time of day, but rather that it also depends on the relative contribution of automatic and controlled processes during encoding. Controlled processes are predominantly involved in encoding attended relative to unattended stimuli. By contrast, automatic processes are predominantly involved in encoding unattended relative to attended stimuli. In non-optimal situations, participants are typically less alert and thus controlled processes cannot be optimally engaged which allows automatic processes to run their course. Hence, irrelevant stimuli are more likely to be encoded (cf., Meier et al., 2003). At test, this benefits performance for previously unattended stimuli. This interpretation is consistent with the notion that automatic and controlled processes are in competition and optimal time of day favours controlled over automatic processes (e.g., May et al., 2005; Yang et al., 2007). 


\section{References}

Anderson, J. A. E., Campbell, K. L., Amer, T., Grady, C. L., \& Hasher, L. (2014). Timing is everything: Age differences in the cognitive control network are modulated by time of day. Psychology and Aging, 29(3), 648-657. http://doi.org/10.1037/a0037243

Baddeley, A. D., Hatter, J. E., Scott, D., \& Snashall, A. (1970). Memory and time of day. Quarterly Journal of Experimental Psychology, 22(4), 605-609. http://doi.org/10.1080/14640747008401939

Blatter, K., \& Cajochen, C. (2007). Circadian rhythms in cognitive performance: Methodological constraints, protocols, theoretical underpinnings. Physiology \& Behavior, 90(2-3), 196-208. http://doi.org/10.1016/j.physbeh.2006.09.009

Graf, P., \& Schacter, D. L. (1985). Implicit and explicit memory for new associations in normal and amnesic subjects. Journal of Experimental Psychology: Learning, Memory, and Cognition, 11(3), 501-518. http://doi.org/http://dx.doi.org.ezproxy.sussex.ac.uk/10.1037/0278-7393.11.3.501

Griefahn, B., Künemund, C., Bröde, P., \& Mehnert, P. (2001). Zur Validität der deutschen Übersetzung des Morningness-Eveningness-Questionnaires von Horne und Östberg. Somnologie, 5(2), 71-80. http://doi.org/10.1046/j.1439-054X.2001.01149.x

Hasher, L., Chung, C., May, C. P., \& Foong, N. (2002). Age, Time of Testing, and Proactive Interference. Canadian Journal of Experimental Psychology, 56(3), 200-207.

Horne, J. A., \& Ostberg, O. (1976). A self-assessment questionnaire to determine morningness-eveningness in human circadian rhythms. International Journal of Chronobiology, 4(2), 97-110.

Intons-Peterson, M. J., Rocchi, P., West, T., McLellan, K., \& Hackney, A. (1998). Aging, optimal testing times, and negative priming. Journal of Experimental Psychology: 
Learning, Memory, and Cognition, 24(2), 362-376.

http://doi.org/http://dx.doi.org/10.1037/0278-7393.24.2.362

May, C. P., Hasher, L., \& Foong, N. (2005). Implicit Memory, Age, and Time of Day. Psychological Science, 16(2), 96-100. http://doi.org/10.1111/j.09567976.2005.00788.x

May, C. P., Hasher, L., \& Stoltzfus, E. R. (1993). Optimal Time of Day and the Magnitude of Age Differences in Memory. Psychological Science, 4(5), 326-330. http://doi.org/10.1111/j.1467-9280.1993.tb00573.x

Meier, B., Morger, V., \& Graf, P. (2003). Competition between automatic and controlled processes. Consciousness and Cognition, 12(2), 309-319. http://doi.org/10.1016/S1053-8100(02)00069-7

Meier, B., Theiler-Bürgi, M., \& Perrig, W. (2009). Levels of Processing and Amnesia Affect Perceptual Priming in Fragmented Picture Naming. International Journal of Neuroscience, 119(8), 1061-1075. http://doi.org/10.1080/00207450802336691

Murray, G., Nicholas, C. L., Kleiman, J., Dwyer, R., Carrington, M. J., Allen, N. B., \& Trinder, J. (2009). Nature's Clocks and Human Mood: The Circadian System Modulates Reward Motivation. Emotion, 9(5), 705-716. http://doi.org/10.1037/a0017080

Paradee, C. V., Rapport, L. J., Hanks, R. A., \& Levy, J. A. (2005). Circadian preference and cognitive functioning among rehabilitation inpatients. Clinical Neuropsychologist, 19(1), 55-72. http://doi.org/10.1080/13854040490524173

Schacter, D. L. (1987). Implicit memory: History and current status. Journal of Experimental Psychology: Learning, Memory, and Cognition, 13(3), 501-518. http://doi.org/10.1037/0278-7393.13.3.501 
Schmidt, C., Collette, F., Cajochen, C., \& Peigneux, P. (2007). A time to think: Circadian rhythms in human cognition. Cognitive Neuropsychology, 24(7), 755-789. http://doi.org/10.1080/02643290701754158

Snodgrass, J. G., \& Vanderwart, M. (1980). A standardized set of 260 pictures: Norms for name agreement, image agreement, familiarity, and visual complexity. Journal of Experimental Psychology: Human Learning, and Memory, 6(2), 174-215.

West, R., Murphy, K. J., Armilio, M. L., Craik, F. I. M., \& Stuss, D. T. (2002). Effects of time of day on age differences in working memory. Journals of Gerontology - Series B Psychological Sciences and Social Sciences, 57(1), P3-P10.

Yang, L., Hasher, L., \& Wilson, D. E. (2007). Synchrony Effects in Automatic and Controlled Retrieval. Psychonomic Bulletin \& Review, 14(1), 51-56. 


\section{Acknowledgements}

N.R. is supported by the Swiss National Science Foundation (Grant PZ00P1_154954).

We would like to thank Mirela Dubravac for her help with the preparation of the stimulus material and the students who helped with data collection.

\section{Declaration of Interest}

The authors report no conflicts of interest. The authors alone are responsible for the content and writing of the paper. 